\title{
HEEGNER POINTS AND JOCHNOWITZ CONGRUENCES ON SHIMURA CURVES
}

\author{
STEFANO VIGNI \\ (Communicated by Matthew A. Papanikolas)
}

\begin{abstract}
Given an elliptic curve $E$ over $\mathbb{Q}$, a suitable imaginary quadratic field $K$ and a quaternionic Hecke eigenform $g$ of weight 2 obtained from $E$ by level raising such that the sign in the functional equation for $L_{K}(E, s)$ (respectively, $\left.L_{K}(g, 1)\right)$ is -1 (respectively, +1 ), we prove a "Jochnowitz congruence" between the algebraic part of $L_{K}^{\prime}(E, 1)$ (expressed in terms of Heegner points on Shimura curves) and the algebraic part of $L_{K}(g, 1)$. This establishes a relation between Zhang's formula of Gross-Zagier type for central derivatives of $L$-series and his formula of Gross type for special values. Our results extend to the context of Shimura curves attached to division quaternion algebras previous results of Bertolini and Darmon for Heegner points on classical modular curves.
\end{abstract}

\section{INTRODUCTION}

The goal of the present article is to use the theory of congruences between modular forms to relate, in the context of elliptic curves, Zhang's formula of Gross-Zagier type for central derivatives of $L$-functions and his formula for special values. This extends previous results of Bertolini and Darmon for Heegner points on modular curves ([5]) to the situation where one needs to work with Shimura curves attached to division quaternion algebras. More precisely, let $E$ be an elliptic curve over $\mathbb{Q}$ of conductor $N=M D$ where $D>1$ is a square-free product of an even number of primes and $(M, D)=1$. By modularity, $E$ is associated with a normalised newform $f=f_{E}$ of weight 2 for $\Gamma_{0}(N)$, whose $q$-expansion will be denoted by

$$
f(q)=\sum_{n \geq 1} a_{n}(f) q^{n}, \quad a_{n}(f) \in \mathbb{Z} .
$$

Let $K$ be an imaginary quadratic field, with ring of integers $\mathcal{O}_{K}$ and discriminant coprime to $N$, in which the primes dividing $M$ (respectively, $D$ ) split (respectively, are inert); in other words, $K$ satisfies a modified Heegner hypothesis relative to $N$. Let $X_{0}^{D}(M)$ be the (compact) Shimura curve over $\mathbb{Q}$ of discriminant $D$ and level $M$, and write $J_{0}^{D}(M)$ for its Jacobian variety. As recalled in $₫ 2.2$, the modularity of $E$ and the Jacquet-Langlands correspondence allow us to introduce a parametrisation

$$
\Pi_{E}: J_{0}^{D}(M) \longrightarrow E
$$

defined over $\mathbb{Q}$. The theory of complex multiplication produces a Heegner divisor class $P_{K} \in J_{0}^{D}(M)(K)$, and we define $\alpha_{K}:=\Pi_{E}\left(P_{K}\right) \in E(K)$ (see \$4.3). The

Received by the editors May 17, 2012 and, in revised form, February 5, 2013.

2010 Mathematics Subject Classification. Primary 11G05, 11G40.

Key words and phrases. Heegner points, Shimura curves, $L$-functions, Jochnowitz congruences. 
Heegner point $\alpha_{K}$ will play a key role in the formulation of our results, which we now briefly describe.

After fixing in 3.1 a suitable "descent prime" $p>3$, in 33.2 we choose a Kolyvagin prime $\ell$ relative to the data $(E, K, p)$. In particular, $\ell$ is inert in $K$ and $p$ divides both $\ell+1$ and $a_{\ell}(f)$. As in [5], the basic idea is to study Hecke congruences modulo $p$ between $f$ and modular forms of level $M \ell$ (or rather, in our situation, between any quaternionic modular form of discriminant $D$ and level $M$ associated with $f$ via the Jacquet-Langlands correspondence and quaternionic modular forms of discriminant $D$ and level $M \ell$ ). To do this, let $X_{0}^{D}(M \ell)$ be the Shimura curve over $\mathbb{Q}$ of discriminant $D$ and level $M \ell$, whose Jacobian we denote $J_{0}^{D}(M \ell)$, and let $\mathbb{T}$ be the Hecke algebra of level $M \ell$ acting on $J_{0}^{D}(M \ell)$. Adopting the usual notation for Hecke operators and writing $-\epsilon$ for the sign in the functional equation for the $L$-function $L(E, s)$ of $E$, we introduce the maximal ideal

$$
\mathfrak{m}:=\left\langle p ; \quad T_{r}-a_{r}(f), r \nmid N \ell ; \quad U_{q}-a_{q}(f), q \mid M ; \quad U_{\ell}-\epsilon\right\rangle
$$

of $\mathbb{T}$ of residual characteristic $p$. Then we denote by $\mathbb{T}_{\mathfrak{m}}$ the completion of $\mathbb{T}$ at $\mathfrak{m}$ and by $I$ the kernel of the natural map $\mathbb{T} \rightarrow \mathbb{T}_{\mathfrak{m}}$. As in 5 , we associate with $\mathfrak{m}$ the quotient $J:=J_{0}^{D}(M \ell) / I J_{0}^{D}(M \ell)$. The abelian variety $J$ is the counterpart in our quaternionic setting of the abelian variety $J^{(\mathfrak{m})}$ introduced by Mazur in [16, Ch. II, $\S 10]$, the main difference being that here, as in [5], the ideal $\mathfrak{m}$ corresponds to an absolutely irreducible mod $p$ Galois representation and hence is not Eisenstein.

In $\$ 3.6$ the Jacquet-Langlands correspondence is combined with level raising results of Diamond-Taylor to prove that $J$ is isogenous to $E^{2} \times J^{\prime}$, where $J^{\prime}$ is a non-zero abelian variety having purely toric reduction at $\ell$. Moreover, the split or non-split nature of this reduction is controlled by $\epsilon$.

If $g$ is a Hecke eigenform of weight 2 on $\Gamma_{0}^{D}(M \ell)$ and $\mathcal{O}_{g}$ is the ring generated by its Hecke eigenvalues, then let $\phi_{g}: \mathbb{T} \rightarrow \mathcal{O}_{g}$ be the (surjective) algebra homomorphism associated with $g$ and set $\mathfrak{m}_{g}:=\phi_{g}(\mathfrak{m})$, which is a maximal ideal of $\mathcal{O}_{g}$ (possibly equal to $\mathcal{O}_{g}$ itself). The eigenform $g$ is said to be a form on $J$ (respectively, $J^{\prime}$ ) if the abelian variety $A_{g}$ attached to $g$ by the Eichler-Shimura construction is a quotient of $J$ (respectively, $J^{\prime}$ ).

Now let $g$ be any form on $J^{\prime}$ and notice that, since $\ell$ is inert in $K$, the sign in the functional equation of $L_{K}(g, s)$ is 1 ; in other words, in passing from level $M$ to level $M \ell$ a change of sign occurs. In light of a formula of Gross (11]) later generalised by Zhang ([22]), in 4.2 we define the algebraic part $\mathbb{L}_{K}(g, 1)$ of the special value $L_{K}(g, 1)$ in terms of optimal embeddings of quadratic orders into Eichler orders of definite quaternion algebras (or, equivalently, in terms of supersingular abelian surfaces in characteristic $\ell$ with quaternionic multiplication). The crucial property of this algebraic part is that $\mathbb{L}_{K}(g, 1)=0$ if and only if $L_{K}(g, 1)=0$.

A simplified form of our main result, which extends [5, Theorem 1.3] and is in fact a corollary of Theorem 4.6, can be stated as follows.

Theorem 1.1. The image of $\alpha_{K}$ in $E\left(K_{\ell}\right) / p E\left(K_{\ell}\right)$ is non-zero if and only if

$$
\mathbb{L}_{K}(g, 1) \not \equiv 0 \quad\left(\bmod \mathfrak{m}_{g}\right)
$$

for all forms $g$ on $J^{\prime}$.

Now write $L_{K}(E, s)$ for the $L$-function of $E$ over $K$. The fact that $K$ satisfies our modified Heegner hypothesis ensures that the sign in the functional equation of $L_{K}(E, s)$ is -1 (see, e.g., [9, Theorem 3.17] for a sketch of a proof in the semistable 
case). By Zhang's formula of Gross-Zagier type ([21, Theorem C]), the point $\alpha_{K}$ encodes the central derivative $L_{K}^{\prime}(E, 1)$; hence Theorem 1.1 can be viewed as providing a congruence modulo $\mathfrak{m}$ between $L_{K}^{\prime}(E, 1)$ and $L_{K}(g, 1)$. Congruences of this type (based on the sign-change phenomenon pointed out above) were first suggested by Jochnowitz (see [14) and then studied and refined by, among others, Bertolini-Darmon and Vatsal ([2], 6], 20]).

As will be apparent later, our strategy to prove Theorem 1.1 is crucially inspired by the arguments in [5]. In fact, since the geometry of the reduction modulo $\ell$ of $X_{0}^{D}(M \ell)$ is analogous to that of the reduction modulo $\ell$ of the modular curve $X_{0}(N \ell)$, such a parallel should come as no surprise. However, various new ingredients are needed in our quaternionic setting. Here we simply highlight the role played by Helm's multiplicity one results for quotients of character groups modulo (non-Eisenstein) maximal ideals of $\mathbb{T}$ that are "controllable at $\ell$ " (see 2.4 ) and our need to use level raising results of Diamond-Taylor in order to show that the abelian variety $J^{\prime}$ is non-trivial (cf. \$3.6). Moreover, the Jacquet-Langlands correspondence between classical and quaternionic modular forms is ubiquitous in our study.

Some consequences of Theorem 1.1 are collected in 44.5. Finally, we remark that [6. Theorem 4.2], which establishes a reciprocity formula for certain classes in the cohomology of elliptic curves over anticyclotomic $\mathbb{Z}_{p}$-extensions, can be regarded as a generalisation of the main result of 5$]$ to an Iwasawa-theoretic context. The line of proof of this result in [6, §9] follows closely the approach to Jochnowitz type congruences proposed in [20] (where these techniques are used to study the nontriviality of Heegner points on classical modular curves; cf. [20, Section 6]), avoiding the analysis of the groups of connected components that appear both here and in [5]. In light of this, Theorems 1.1 and 4.6 can be viewed as explicit incarnations in the language of [5] - of some of the ideas underlying the theory developed in [6].

\section{Background on Shimura Curves and Hecke Algebras}

2.1. Degeneracy maps and Hecke algebras. Let $B$ be the quaternion algebra over $\mathbb{Q}$ of discriminant $D$ and fix once and for all an isomorphism of $\mathbb{R}$-algebras

$$
i_{\infty}: B \otimes_{\mathbb{Q}} \mathbb{R} \cong \mathcal{M}_{2}(\mathbb{R})
$$

which exists because $B$ is indefinite (i.e., splits at the archimedean place of $\mathbb{Q}$ ). Let $\ell$ be a prime not dividing $N$, let $R(M \ell) \subset R(M)$ be Eichler orders of $B$ of level $M \ell$ and $M$, and let $\Gamma_{0}^{D}(M \ell) \subset \Gamma_{0}^{D}(M)$ be the corresponding groups of norm 1 elements, so that

$$
X_{0}^{D}(M)=\Gamma_{0}^{D}(M) \backslash \mathcal{H}, \quad X_{0}^{D}(M \ell)=\Gamma_{0}^{D}(M \ell) \backslash \mathcal{H}
$$

as Riemann surfaces. There are two natural degeneracy maps

$$
\pi_{1}, \pi_{2}: X_{0}^{D}(M \ell) \longrightarrow X_{0}^{D}(M),
$$

induced by the identity and the multiplication by $\omega_{\ell}$ on $\mathcal{H}$, respectively, where $\omega_{\ell} \in$ $R(M \ell)$ has reduced norm $\ell$ (such an element normalises $\Gamma_{0}^{D}(M \ell)$ ). By covariant and contravariant functoriality, these degeneracy maps induce maps

$$
\pi_{1, *}, \pi_{2, *}: J_{0}^{D}(M \ell) \longrightarrow J_{0}^{D}(M), \quad \pi_{1}^{*}, \pi_{2}^{*}: J_{0}^{D}(M) \longrightarrow J_{0}^{D}(M \ell)
$$

between Jacobian varieties. 
For any integer $S \geq 1$ coprime to $D$, write $\mathbb{T}(S)$ for the Hecke algebra of level $S$, i.e., the subring of the endomorphism ring of $J_{0}^{D}(S)$ generated over $\mathbb{Z}$ by the Hecke operators $T_{q}$ with $q \nmid S D$ and $U_{q}$ with $q \mid S$. Then the degeneracy maps $\pi_{1}$ and $\pi_{2}$ satisfy the relations

$$
\pi_{1, *} \circ \pi_{1}^{*}=\pi_{2, *} \circ \pi_{2}^{*}=\ell+1, \quad \pi_{2, *} \circ \pi_{1}^{*}=\pi_{1, *} \circ \pi_{2}^{*}=T_{\ell} .
$$

Define an acton of $\mathbb{T}:=\mathbb{T}(M \ell)$ on $J_{0}^{D}(M)^{2}$ by letting the Hecke operators $T_{q}$ and $U_{q}$ for $q \neq \ell$ act diagonally and by letting $U_{\ell}$ act by left multiplication by the matrix $\left(\begin{array}{cc}T_{\ell} & \ell \\ -1 & 0\end{array}\right)$. Then set

$$
\pi^{*}:=\pi_{1}^{*} \oplus \pi_{2}^{*}: J_{0}^{D}(M)^{2} \rightarrow J_{0}^{D}(M \ell), \pi_{*}:=\left(\pi_{1, *}, \pi_{2, *}\right): J_{0}^{D}(M \ell) \rightarrow J_{0}^{D}(M)^{2} .
$$

The following result is proved as [5. Lemma 2.1] (for a precise reference in the case of our interest, see [13, p. 93]).

Lemma 2.1. The maps $\pi^{*}$ and $\tilde{\pi}_{*}$ are compatible with the actions of $\mathbb{T}$ on $J_{0}^{D}(M \ell)$ and $J_{0}^{D}(M)^{2}$ defined above.

2.2. Jacquet-Langlands and modularity. Let $S_{2}\left(\Gamma_{0}^{D}(M)\right)$ denote the $\mathbb{C}$-vector space of modular forms of weight 2 on $\Gamma_{0}^{D}(M)$ (see $[9, \S 4.2]$ ) and recall that $N=M D$. The Jacquet-Langlands correspondence (see, e.g., [9, §4.5] and [13, Theorem 2.3]) gives a non-canonical isomorphism between $S_{2}^{D \text {-new }}\left(\Gamma_{0}(N)\right)$ and $S_{2}\left(\Gamma_{0}^{D}(M)\right)$ induced by a natural isomorphism at the level of Hecke algebras. Fix a form $f^{\mathrm{JL}} \in$ $S_{2}\left(\Gamma_{0}^{D}(M)\right)$ associated with $f$ via the Jacquet-Langlands correspondence; then $f^{\mathrm{JL}}$ is uniquely determined up to multiplication by elements in $\mathbb{C}^{\times}$. With notation as in (11), the form $f^{\mathrm{JL}}$ has the same Hecke eigenvalues as $f$; more precisely,

$$
T_{r}\left(f^{\mathrm{JL}}\right)=a_{r}(f) f^{\mathrm{JL}}, \quad U_{q}\left(f^{\mathrm{JL}}\right)=a_{q}(f) f^{\mathrm{JL}}, \quad W_{t}\left(f^{\mathrm{JL}}\right)=a_{t}(f) f^{\mathrm{JL}}
$$

for all primes $r \nmid N$, all primes $q \mid M$ and all primes $t \mid D$, where $W_{t}$ is the AtkinLehner involution defined in [2, p. 422].

Following [21] and [22], use the Hodge class in $\operatorname{Pic}\left(X_{0}^{D}(M)\right) \otimes \mathbb{Q}($ see $[8, \S 3.5])$ to introduce a finite map $\iota_{M}: X_{0}^{D}(M) \rightarrow J_{0}^{D}(M)$ defined over $\mathbb{Q}$. Thanks to the modularity of $E$, the Jacquet-Langlands correspondence and Faltings's isogeny theorem (see [9, §4.6] and [21, §3.4.4]), this map can in turn be used to obtain a surjective morphism $\pi_{E}: X_{0}^{D}(M) \rightarrow E$ defined over $\mathbb{Q}$, which we fix. The map $\pi_{E}$ induces two maps

$$
\Pi_{E}:=\pi_{E, *}: J_{0}^{D}(M) \longrightarrow E, \quad \pi_{E}^{*}: E \longrightarrow J_{0}^{D}(M),
$$

by Albanese (i.e., covariant) and Picard (i.e., contravariant) functoriality, respectively. At the cost of replacing $E$ with an isogenous curve, from now on we shall always assume that $E$ is a strong Weil curve and that $\pi_{E}$ is a strong Weil parametrisation, in the sense that $\pi_{E, *}$ has a connected kernel (or, equivalently, that $\pi_{E}^{*}$ is injective).

2.3. Enhanced QM surfaces and character groups of Jacobians. In what follows let $B$ be the indefinite quaternion algebra over $\mathbb{Q}$ of discriminant $D$ introduced in $\$ 2.1$ and let $\mathcal{B}$ be the definite quaternion algebra over $\mathbb{Q}$ of discriminant $D \ell$ : the interplay between $B$ and $\mathcal{B}$ lies at the core of our subsequent considerations.

We refer the reader to [4, §1] for the notion of orientation of an Eichler order. Let $R_{1}, \ldots, R_{t}$ be representatives for the conjugacy classes of oriented Eichler orders of level $M$ in $\mathcal{B}$; we denote their classes $\left[R_{i}\right]$ and set $\mathcal{E}:=\left\{\left[R_{1}\right], \ldots,\left[R_{t}\right]\right\}$. Let $\mathscr{M}:=\mathbb{Z}[\mathcal{E}]$ be the free abelian group over $\mathcal{E}$. We want to describe a geometric 
interpretation of $\mathscr{M}$ in terms of abelian surfaces with quaternionic multiplication (QM surfaces, for short), whose definition is recalled, e.g., in [4, §4].

Definition 2.2. An enhanced $Q M$ surface with $M$-level structure over a field $k$ is a pair $(A, C)$, where $A$ is a QM surface over $k$ and $C \subset A$ is a $k$-rational subgroup of order $M$ whose points over the algebraic closure of $k$ form a cyclic group.

When $k$ is a finite extension of $\mathbb{F}_{\ell}$ we say that $(A, C)$ is an enhanced QM surface in characteristic $\ell$. There is an evident notion of isomorphism between such enhanced QM surfaces. As in [4, §5], write $\underline{\operatorname{End}}(A)$ for the endomorphism ring of the pair $(A, C)$. It is a basic fact that if $(A, C)$ is an enhanced QM surface with $M$-level structure in characteristic $\ell$, then $\operatorname{End}(A)$ is (isomorphic to) either an order in an imaginary quadratic field or an Eichler order of level $M$ in $\mathcal{B}$ (cf. [7, §3.2] and [18, Theorem 4.2.1]).

Definition 2.3. An enhanced QM surface $(A, C)$ with $M$-level structure in characteristic $\ell$ is supersingular if $\underline{\operatorname{End}}(A)$ is an Eichler order in $\mathcal{B}$.

The following result is a generalisation of [17, Proposition 3.3].

Proposition 2.4. The set of isomorphism classes of supersingular QM surfaces in characteristic $\ell$ is in bijection with the set of oriented Eichler orders of level $M$ in $\mathcal{B}$.

Proof. The bijection sends the class $[(A, C)]$ to the Eichler order End $(A)$, which is oriented as in [18, Remark 1.2.4]. For details, see [18.

Write $\mathcal{X}_{0}^{D}(M)$ for Drinfeld's model of $X_{0}^{D}(M)$ over $\mathbb{Z}([7, \S 3.1],[18, \S 4.1])$ and let $\mathcal{X}:=\mathcal{X}_{0}^{D}(M) \times_{\mathbb{Z}} \mathbb{Z}_{\ell}$ be its base change to $\mathbb{Z}_{\ell}$. Since $\ell \nmid N$, the special fibre $\tilde{\mathcal{X}}$ of $\mathcal{X}$ is non-singular $([7, \S 3.2],[18, \S 4.2])$ and its points over $\overline{\mathbb{F}}_{\ell}$ correspond to classes of enhanced QM surfaces in characteristic $\ell$. We say that $[(A, C)] \in \tilde{\mathcal{X}}_{/ \overline{\mathbb{F}}_{\ell}}$ is supersingular if $(A, C)$ is supersingular according to Definition 2.3

Let $\Sigma$ be the set of supersingular points of $\tilde{\mathcal{X}}_{/ \overline{\mathbb{F}}_{\ell}}$. We immediately get

Proposition 2.5. The sets $\Sigma$ and $\mathcal{E}$ are in bijection.

Now let $\mathcal{J}_{0}^{D}(M \ell)$ be the Néron model of $J_{0}^{D}(M \ell)$ over $\mathbb{Z}_{\ell}$ and let $\mathcal{J}_{0}^{D}(M \ell)^{0}$ be its identity component. The special fibre of $\mathcal{J}_{0}^{D}(M \ell)^{0}$ is an extension of the abelian variety $J_{0}^{D}(M)^{2}$ over $\mathbb{F}_{\ell}$ by a torus $T$. The character group $X^{*}(T)$ of $T$ is a free abelian group of finite rank that inherits an action of the Hecke algebra $\mathbb{T}$. In fact, results of Grothendieck and Raynaud ensure that $X^{*}(T)$ is isomorphic to the group $\mathbb{Z}[\Sigma]^{0}$ of degree zero divisors on $\Sigma$. Notice that, by Proposition $2.5, \mathbb{Z}[\Sigma]$ is naturally identified with $\mathscr{M}$.

2.4. Component groups and multiplicity one. Let $F$ be a finite extension of $\mathbb{Q}_{\ell}$ with ring of integers $\mathcal{O}_{F}$ and ramification index $e=e_{F}$, and let $\mathcal{J}_{0}^{D}(M \ell)_{F}$ denote the Néron model of $J_{0}^{D}(M \ell)$ over $\mathcal{O}_{F}$. The group $\Phi\left(J_{0}^{D}(M \ell)_{/ F}\right)$ of connected components of the special fibre of $\mathcal{J}_{0}^{D}(M \ell)_{F}$ fits into a short exact sequence

$$
0 \longrightarrow X^{*}(T) \otimes(\mathbb{Z} / e \mathbb{Z}) \longrightarrow \Phi\left(J_{0}^{D}(M \ell)_{/ F}\right) \longrightarrow \Phi\left(J_{0}^{D}(M \ell)_{/ \mathbb{Q}_{\ell}}\right) \longrightarrow 0,
$$

the last map being induced from the norm if the extension $F / \mathbb{Q}_{\ell}$ is totally ramified. The Hecke module $\Phi\left(J_{0}^{D}(M \ell) / \mathbb{Q}_{\ell}\right)$ is Eisenstein (see [13, p. 95]); that is, its support consists exclusively of maximal ideals $\mathfrak{m}$ of $\mathbb{T}$ that are Eisenstein in the sense that their associated residual Galois representations $\bar{\rho}_{\mathfrak{m}}$ fail to be absolutely irreducible. In particular, we obtain 
Proposition 2.6. The completion of $\Phi\left(J_{0}^{D}(M \ell)_{/ F}\right)$ at a non-Eisenstein maximal ideal $\mathfrak{m}$ of $\mathbb{T}$ is isomorphic as a Hecke module to the completion of $X^{*}(T) \otimes(\mathbb{Z} / e \mathbb{Z})$ at $\mathfrak{m}$.

The following notion was introduced by Helm ([13, Definition 6.4]).

Definition 2.7. Let $\mathfrak{m}$ be a non-Eisenstein maximal ideal of $\mathbb{T}$ of residual characteristic $p$. Then $\mathfrak{m}$ is controllable at $\ell$ if one of the following holds:

(1) $\bar{\rho}_{\mathfrak{m}}$ is not finite at $\ell$;

(2) $\bar{\rho}_{\mathfrak{m}}$ is unramified at $\ell, \ell \neq p$ and $\bar{\rho}_{\mathfrak{m}}\left(\right.$ Frob $\left._{\ell}\right)$ is not a scalar;

(3) $\ell=p$ and $p \neq 2$;

(4) $\ell=p=2$ and the restriction of $\bar{\rho}_{\mathfrak{m}}$ to a decomposition group at 2 is not contained in the scalar matrices.

The multiplicity one result below is the counterpart of [17, Theorem 6.4].

Theorem 2.8 (Helm). Suppose that $\mathfrak{m}$ is a maximal ideal of $\mathbb{T}$ that is controllable at $\ell$. Then the quotient $X^{*}(T) / \mathfrak{m} X^{*}(T)$ is a one-dimensional vector space over $\mathbb{T} / \mathfrak{m}$.

Proof. This is [13, Lemma 6.5].

\section{A DISTINGUISHED ABELIAN VARIETY}

3.1. The auxiliary prime $p$. Write $\mathcal{I}=\mathcal{I}(E)$ for the isogeny class of $E$ and let $m(\mathcal{I})$ be the degree of a (classical) parametrisation $X_{0}(N) \rightarrow E^{\prime}$ with $E^{\prime} \in \mathcal{I}$ and the kernel of the surjection $J_{0}(N) \rightarrow E^{\prime}$ connected (note that $E^{\prime}$ generally differs from $E$ ). Let us fix a "descent prime" $p$ such that

(1) the $\bmod p$ Galois representation $\bar{\rho}_{E, p}$ attached to $E$ is absolutely irreducible;

(2) $p \nmid 6 N m(\mathcal{I})$.

Serre's "open image theorem" ([19]) guarantees that all but finitely many primes $p$ satisfy these two conditions.

3.2. Choice of $\ell$ and level raising. Let $p$ be as in 3.1 let $E[p]$ be the group of $p$-torsion points of $E$ and write $K(E[p])$ for the smallest extension of $K$ containing the coordinates of these points. Moreover, let $\delta_{K}$ be the discriminant of $K$. Recall from [5, p. 261] that a prime $\ell$ is called a Kolyvagin prime (relative to $E, K$ and $p)$ if

(1) $\ell \nmid N \delta_{K} p$ (so that $\ell$ is unramified in $K(E[p])$ );

(2) Frob $_{\ell} \in \operatorname{Gal}(K(E[p]) / \mathbb{Q})$ belongs to the conjugacy class of complex conjugation.

In particular, $\ell$ is inert in $K$ and $p$ divides both $\ell+1$ and $a_{\ell}(f)$. By Čebotarev's density theorem, there are infinitely many Kolyvagin primes relative to $(E, K, p)$. Note that every Kolyvagin prime $\ell$ satisfies the claims of [13, Lemma 7.1] (see the proof of [13, Lemma 7.1]).

Fix a Kolyvagin prime $\ell$ relative to $(E, K, p)$ and, as before, write $\mathbb{T}$ for the Hecke algebra $\mathbb{T}(M \ell)$ of level $M \ell$. As in [5], our goal is to study certain modular forms of level $M \ell$ that are congruent to $f$ (or, rather, to $f^{\mathrm{JL}}$ ) modulo $p$. Recall from the introduction that $-\epsilon$ is the sign in the functional equation for $L(E, s)$ and define

$$
\mathfrak{m}:=\left\langle p ; \quad T_{r}-a_{r}(f), r \nmid N \ell ; \quad U_{q}-a_{q}(f), q \mid M ; \quad U_{\ell}-\epsilon\right\rangle .
$$


If $M$ is a $\mathbb{T}$-module, then let $M_{\mathfrak{m}}$ denote the completion of $M$ at $\mathfrak{m}$; the ideal $\mathfrak{m}$ is said to be in the support of $M$ if $M_{\mathfrak{m}} \neq 0$. Let $I$ be the kernel of the natural map $\mathbb{T} \rightarrow \mathbb{T}_{\mathfrak{m}}$.

In Definition 2.7 we explained the notion of controllability for (non-Eisenstein) maximal ideals of $\mathbb{T}$; here we record the following

Lemma 3.1. The ideal $\mathfrak{m}$ is controllable at $\ell$.

Proof. First of all, the Galois representation $\bar{\rho}_{\mathfrak{m}}$ is isomorphic to $\bar{\rho}_{E, p}$, so it is absolutely irreducible by assumption (1) made on $p$ in 3.1 . Therefore $\mathfrak{m}$ is nonEisenstein. On the other hand, $\ell$ is a Kolyvagin prime, which means that Frob $\ell \in$ $\operatorname{Gal}(K(E[p]) / \mathbb{Q})$ belongs to the conjugacy class of complex conjugation. Since complex conjugation acts on $E[p]$ with eigenvalues \pm 1 , it follows that $\bar{\rho}_{\mathfrak{m}}\left(\right.$ Frob $\left._{\ell}\right)$ is not a scalar.

Finally, thanks to the functoriality of the Hodge class (i.e., the natural compatibility of Hodge classes at levels $M$ and $M \ell$ under pull-back and push-forward via the degeneracy map $\pi_{1}$ considered in (3); see [8, §3.5]), we define maps $\iota_{M}$ and $\iota_{M \ell}$ as in $\$ 2.2$ in such a way that the square

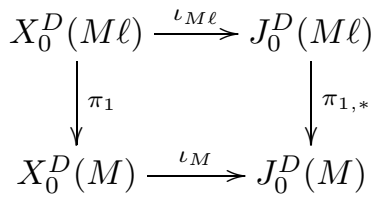

is commutative.

3.3. The modular form $f_{\ell}$. The cusp form $f \in S_{2}\left(\Gamma_{0}(N)\right)$ associated with $E$ is not an eigenform for the Hecke algebra of level $N \ell$, because it fails to be an eigenform for the Hecke operator at $\ell$. As in [5, p. 267], choose a root $\beta$ of the polynomial $X^{2}-a_{\ell}(f) X+\ell$ and define the (classical) modular form $f_{\ell}(z):=$ $f(z)-\ell / \beta f(\ell z)$ with coefficients in the imaginary quadratic order $\mathbb{Z}[\beta]$. Then $f_{\ell}$ is an eigenform of level $N \ell$ in the same old-class as $f$ and with eigenvalue $\beta$ at $\ell$.

Fix a form $f_{\ell}^{\mathrm{JL}} \in S_{2}\left(\Gamma_{0}^{D}(M \ell)\right)$ corresponding, as in $\$ 2.2$, to $f_{\ell}$ under the JacquetLanglands correspondence and let $\mathfrak{m}_{f} \mathrm{JL}$ be the ideal $(p, \beta-\epsilon)$ of $\mathbb{Z}[\beta]$. Then the maximal ideal $\mathfrak{m}$ of $\$ 3.2$ is the inverse image of $\mathfrak{m}_{f} \mathrm{sL}$ under the homomorphism $\mathbb{T} \rightarrow \mathbb{Z}[\beta]$ determined by $f_{\ell}^{\mathrm{JL}}$.

3.4. The abelian variety $J$. Let $I \subset \mathbb{T}$ be as in $₫ 3.2$ and consider the abelian variety

$$
J:=J_{0}^{D}(M \ell) / I J_{0}^{D}(M \ell) .
$$

If $g$ is a Hecke eigenform of weight 2 on $\Gamma_{0}^{D}(M \ell)$, so that $g$ corresponds to a onedimensional Hecke-stable subspace of $S_{2}^{D \text {-new }}\left(\Gamma_{0}(N \ell)\right)$ by Jacquet-Langlands, and $\mathcal{O}_{g}$ is the ring generated by its Hecke eigenvalues, then let $\phi_{g}: \mathbb{T} \rightarrow \mathcal{O}_{g}$ be the (surjective) algebra homomorphism associated with $g$ (cf. [2, Theorem 1.2]), whose kernel we denote by $I_{g}$.

Definition 3.2. The form $g$ is a form on $J$ if the following equivalent conditions hold:

(1) the abelian variety $A_{g}:=J_{0}^{D}(M \ell) / I_{g} J_{0}^{D}(M \ell)$ is a quotient of $J$;

(2) $I \subset I_{g}$; 
(3) the ideal $\mathfrak{m}_{g}:=\phi_{g}(\mathfrak{m})$ is a proper maximal ideal of $\mathcal{O}_{g}$ and

$$
a_{n}\left(f_{\ell}^{\mathrm{JL}}\right) \quad\left(\bmod \mathfrak{m}_{f} \mathrm{JL}\right)=a_{n}(g) \quad\left(\bmod \mathfrak{m}_{g}\right)
$$

for all integers $n \geq 1$ coprime to $D$.

There is only one $\ell$-old form (up to the Galois action) that is congruent to $f_{\ell}^{\mathrm{JL}}$ modulo $p$ (that is, the Hecke eigenvalues are congruent modulo $p$ ), namely $f_{\ell}^{\mathrm{JL}}$ itself. This holds because, since $p \nmid N m(\mathcal{I})$ (cf. 33.1), $p$ is not a congruence prime for $E$ (see [1, Theorem 2.1]). Moreover, the abelian variety $A_{f_{\ell}^{\mathrm{JL}}}=J_{0}^{D}(M \ell) / I_{f_{\ell}^{\mathrm{JL}}} J_{0}^{D}(M \ell)$ is isogenous to $E \times E$; this can be deduced from a comparison of the $L$-functions of $A_{f_{\ell}^{\mathrm{JL}}}$ and of $E \times E$ and an application of Faltings's isogeny theorem (cf. [21, §3.4.4] for computations in the same spirit). As a consequence, there is an isogeny

$$
J \sim E^{2} \times \prod_{[g]} A_{g}
$$

where the product is taken over the Galois orbits of the eigenforms on $J$ that are new at $\ell$, in the sense that the classical cusp forms associated with them by the Jacquet-Langlands correspondence are new at $\ell$.

As in 5], we describe such an isogeny. Consider the composition

$$
\varphi_{E}: E^{2} \stackrel{\pi_{E}^{*}}{\longrightarrow} J_{0}^{D}(M)^{2} \stackrel{\pi^{*}}{\longrightarrow} J_{0}^{D}(M \ell) \stackrel{\pi_{J}}{\longrightarrow} J,
$$

where $\pi^{*}$ is as in $\$ 2.1$ and $\pi_{J}$ is the canonical projection. Thanks to Lemma 2.1, the map $\varphi_{E}$ is $\mathbb{T}$-equivariant with respect to the action of $\mathbb{T}$ on $E^{2}$ defined by letting $T_{r}$ for $r \nmid N \ell$ and $U_{r}$ for $r \mid M$ act by multiplication by $a_{r}(f)$ and letting $U_{\ell}$ act by left mutiplication by the matrix $\left(\begin{array}{cc}a_{\ell}(f) & \ell \\ -1 & 0\end{array}\right)$.

3.5. The $\ell$-new subvariety and its reduction. Let $J_{0}^{D}(M \ell)^{\ell \text {-new }}$ be the $\ell$-new subvariety of $J_{0}^{D}(M \ell)$, that is, the kernel of the map $\pi_{*}$ introduced in $\$ 2.1$. The following result is well known to the experts. Thanks to the reduction properties of $X_{0}^{D}(M \ell)$ at $\ell([7$, Theorem 13], [18, $\S 4.3])$, its proof proceeds as in the case of modular Jacobians; see [15, Proposition 1].

Proposition 3.3. The abelian variety $J_{0}^{D}(M \ell)^{\ell-n e w}$ has purely toric reduction at $\ell$; it is the maximal toric subvariety of $J_{0}^{D}(M \ell)$ at $\ell$.

3.6. The abelian variety $J^{\prime}$. Write $J^{\prime}$ for the image of $J_{0}^{D}(M \ell)^{\ell \text {-new }}$ in $J$ and let $\varphi^{\prime}: J^{\prime} \hookrightarrow J$ be the natural inclusion. Then define an isogeny $\varphi$ as

$$
\varphi:=\varphi_{E}+\varphi^{\prime}: E^{2} \times J^{\prime} \longrightarrow J .
$$

The following result is the analogue of [5, Proposition 3.1]. Note that, in our quaternionic context, it is necessary to invoke general level raising results of Diamond-Taylor ([10]) and not just those originally due to Ribet.

Proposition 3.4. The abelian variety $J^{\prime}$ is not trivial and has purely toric reduction at $\ell$. This reduction is split if $\epsilon=1$ and is non-split if $\epsilon=-1$.

Proof. By (6) and Poincaré's complete reducibility theorem, there is an isogeny $J^{\prime} \sim \prod_{[g]} A_{g}$, where the product is over the Galois orbits of the eigenforms on $J$ that are new at $\ell$. These are weight 2 eigenforms $g$ on $\Gamma_{0}^{D}(M \ell)$ that are new at $\ell$, satisfy $\mathfrak{m}_{g} \neq \mathcal{O}_{g}$ and such that the congruence

$$
a_{n}\left(f_{\ell}^{\mathrm{JL}}\right) \quad\left(\bmod \mathfrak{m}_{f} \mathrm{JL}\right)=a_{n}(g) \quad\left(\bmod \mathfrak{m}_{g}\right)
$$


holds for all $n \geq 1$ coprime to $D$ (cf. Definition 3.2). Thanks to our choice of $\ell$, level raising results of Diamond-Taylor ([10]) ensure that there exists a form $g$ that is new at $\ell$ and satisfies (8) (see, e.g., [13, Lemma 7.1] for a precise statement). It follows that the abelian variety $J^{\prime}$ is not trivial. As for the part about reduction, $J^{\prime}$ is (isomorphic to) a quotient of $J_{0}^{D}(M \ell)^{\ell-\text { new }}$; hence Proposition 3.3 implies that $J^{\prime}$ has purely toric reduction at $\ell$. Finally, with $g$ being any form as above, it is known that $a_{\ell}(g)=1$ (respectively, $a_{\ell}(g)=-1$ ) if and only if $A_{g}$ has split (respectively, non-split) multiplicative reduction at $\ell$. But $a_{\ell}(g) \equiv \epsilon\left(\bmod \mathfrak{m}_{g}\right)$ and $p \neq 2$, and the proposition is proved.

Proposition 3.4 allows us to apply to $J^{\prime}$ the Tate-Mumford theory of nonarchimedean ( $\ell$-adic) uniformisation of abelian varieties with purely toric reduction. In particular, we shall use [5, Corollary 3.2].

Lemma 3.5. The ideal $\mathfrak{m}$ is not in the support of the kernel of $\varphi_{E}$.

Proof. By the results in [10] (see, in particular, [10, §3]), the support in $\mathbb{T}$ of the kernel of $\pi^{*}$ consists entirely of Eisenstein maximal ideals, and then one can proceed as in the proof of [5, Lemma 3.3].

3.7. An analysis of component groups. In analogy with what was done in 2.4 for $J_{0}^{D}(M \ell)$, if $F$ is a finite field extension of $K_{\ell}$ with ring of integers $\mathcal{O}_{F}$, then denote by $\mathcal{J}_{F}$ (respectively, $\mathcal{J}_{F}^{\prime}$ ) the Néron model of $J$ (respectively, $J^{\prime}$ ) over $\mathcal{O}_{F}$, and write $\Phi\left(J_{/ F}\right)$ (respectively, $\Phi\left(J_{/ F}^{\prime}\right)$ ) for the component group of this Néron model. By a slight abuse of notation, we shall also write $J_{0}^{D}(M \ell)(F)$ in place of $\mathcal{J}_{0}^{D}(M \ell)\left(\mathcal{O}_{F}\right)$, and similarly for $J$ and $J^{\prime}$.

Since most of the arguments used in [5, §3] for modular Jacobians carry over mutatis mutandis to our setting, we refer to 5 whenever convenient.

If $M$ is a module on which complex conjugation in $G_{\mathbb{Q}}$ acts, then write $M^{ \pm}$for the submodules of $M$ on which this involution acts as \pm 1 .

Proposition 3.6. The map

$$
\boldsymbol{i}: E^{2}\left(K_{\ell}\right)_{\mathfrak{m}}^{\epsilon} \longrightarrow J\left(K_{\ell}\right)_{\mathfrak{m}}^{\epsilon}
$$

induced by $\varphi_{E}$ is an isomorphism.

Proof. Combine Lemma 3.5 with a study of the kernel of the isogeny $\varphi$ introduced in (7), as in the proof of [5, Proposition 3.7].

Let $H$ be the Hilbert class field of $K$ and let $\mathcal{L}$ be the ring class field of $K$ of conductor $\ell$. Every prime of $H$ above $\ell$ is totally ramified in $\mathcal{L}$, and we write $\mathcal{L}_{\ell}$ for the completion of $\mathcal{L}$ at any such prime above $\ell$; then $\mathcal{L}_{\ell}$ is a totally ramified cyclic extension of $K_{\ell}$ of degree $(\ell+1) / u$ where $u:=\# \mathcal{O}_{K}^{\times} / 2$. As in [5, p. 274], let us introduce the group of local points

$$
\tilde{J}:=\frac{J\left(\mathcal{L}_{\ell}\right)}{\varphi_{E}\left(E^{2}\left(\mathcal{L}_{\ell}\right)\right)+(\sigma-1) J\left(\mathcal{L}_{\ell}\right)} .
$$

The module $\tilde{J}$ is endowed with a Hecke action and with an action of complex conjugation. Since $\varphi_{E}\left(E^{2}\right)$ is contained in the identity component of $J$ and $\operatorname{Gal}\left(\mathcal{L}_{\ell} / K_{\ell}\right)$ acts trivially on $\Phi\left(J_{/ \mathcal{L}_{\ell}}\right)$ (because it acts trivially on $\Phi\left(J_{0}^{D}(M \ell) / \mathcal{L}_{\ell}\right)$ ), projection onto the group of components gives a map

$$
\boldsymbol{p}: \tilde{J}_{\mathfrak{m}}^{\epsilon} \longrightarrow \Phi\left(J_{/ \mathcal{L}_{\ell}}\right)_{\mathfrak{m}} .
$$


Proposition 3.7. The map $\boldsymbol{p}$ is an isomorphism.

Proof. The surjectivity of $\boldsymbol{p}$ is clear. To show injectivity one can apply [5, Corollary 3.2] and argue exactly as in the proof of [5, Proposition 3.8].

Corollary 3.8. The group $(\tilde{J} / \mathfrak{m} \tilde{J})^{\epsilon}$ is one-dimensional over $\mathbb{T} / \mathfrak{m}$.

Proof. The quotient $\Phi\left(J_{/ \mathcal{L}_{\ell}}\right) / \mathfrak{m} \Phi\left(J_{/ \mathcal{L}_{\ell}}\right)$ is one-dimensional over $\mathbb{T} / \mathfrak{m}$ by a combination of Theorem 2.8 and Proposition 2.6, and then the corollary follows from Proposition 3.7 .

Fix a generator $\sigma$ of $\operatorname{Gal}\left(\mathcal{L}_{\ell} / K_{\ell}\right)=\operatorname{Gal}(\mathcal{L} / H)$ and write $N_{\mathcal{L}_{\ell} / K_{\ell}}:=\sum_{i=1}^{(\ell+1) / u} \sigma^{i}$ for the norm map of $\mathcal{L}_{\ell}$ over $K_{\ell}$. Since $(\ell+1) / u$ is prime to $\ell$ and the residual characteristic $p$ of $\mathfrak{m}$ divides $\ell+1$ (cf. $§ 3.2)$, it follows that $N_{\mathcal{L}_{\ell} / K_{\ell}}$ induces a well-defined map

$$
\boldsymbol{n}: \tilde{J} / \mathfrak{m} \tilde{J} \longrightarrow J\left(K_{\ell}\right) / \mathfrak{m} J\left(K_{\ell}\right)
$$

that is equivariant for the action of complex conjugation (see [5, p. 275]).

Proposition 3.9. The induced map

$$
\boldsymbol{n}:(\tilde{J} / \mathfrak{m} \tilde{J})^{\epsilon} \longrightarrow\left(J\left(K_{\ell}\right) / \mathfrak{m} J\left(K_{\ell}\right)\right)^{\epsilon}
$$

is an isomorphism.

Proof. As in the proof of [5, Proposition 3.10] (to which we refer for details), one can reduce the surjectivity of $\boldsymbol{n}$ to a statement in local class field theory for the extension $\mathcal{L}_{\ell} / K_{\ell}$ which is true by [5, Corollary 3.2]. Finally, to prove that $\boldsymbol{n}$ is injective observe that, by Corollary $\left[3.8,(\tilde{J} / \mathfrak{m} \tilde{J})^{\epsilon}\right.$ is a one-dimensional $\mathbb{F}_{p}$-vector space and that the same is true of $\left(J\left(K_{\ell}\right) / \mathfrak{m} J\left(K_{\ell}\right)\right)^{\epsilon}$ by Proposition 3.6 (cf. the proof of [5, Lemma 3.4] for details).

By an abuse of notation, write $\boldsymbol{i}$ and $\boldsymbol{p}$ for the isomorphisms between $\bmod \mathfrak{m}$ quotients induced by the maps in Propositions 3.6 and 3.7 By an analogue of [5. Lemma 3.5], there is an isomorphism

$$
j: \Phi\left(J_{0}^{D}(M \ell)_{/ \mathcal{L}_{\ell}}\right) / \mathfrak{m} \stackrel{\cong}{\longrightarrow} \Phi\left(J_{/ \mathcal{L}_{\ell}}\right) / \mathfrak{m} .
$$

Since the ideal $\mathfrak{m}$ is not Eisenstein and $X^{*}(T)=\mathscr{M}^{0}$ canonically, Proposition 2.6 gives an identification $\Phi\left(J_{0}^{D}(M \ell) / \mathcal{L}_{\ell}\right) / \mathfrak{m}=\mathscr{M}^{0} / \mathfrak{m} \mathscr{M}^{0}$. Then, as in [5, p. 276], we define the isomorphism of one-dimensional $\mathbb{F}_{p}$-vector spaces

$$
\eta:=\boldsymbol{j}^{-1} \circ \boldsymbol{p} \circ \boldsymbol{n}^{-1} \circ \boldsymbol{i}:\left(E^{2}\left(K_{\ell}\right) / \mathfrak{m} E^{2}\left(K_{\ell}\right)\right)^{\epsilon} \stackrel{\cong}{\longrightarrow} \mathscr{M}^{0} / \mathfrak{m} \mathscr{M}^{0} .
$$

This map will play an important role in our subsequent arguments.

\section{Special VAlues And Jochnowitz CONGRUEnCES}

4.1. The module $\mathscr{M}_{g}^{0}$. Recall from $₫ 2.3$ that $\mathscr{M}=\mathbb{Z}[\mathcal{E}]$, the free abelian group over the (finite) set $\mathcal{E}$ of conjugacy classes of oriented Eichler orders of level $M$ in $\mathcal{B}$. Then $X^{*}(T)$ can be identified with the subgroup $\mathscr{M}^{0}$ of degree zero divisors in $\mathscr{M}$. The module $\mathscr{M}$ is endowed with a natural Hecke action compatible with the inclusion $\mathscr{M}^{0} \subset \mathscr{M}$.

Notation being as in $₫ 3.4$, if $g$ is a form on $J^{\prime}$, then let $\mathcal{O}_{g, \mathfrak{m}}$ be the completion of $\mathcal{O}_{g}$ at $\mathfrak{m}_{g}$. Moreover, set

$$
\mathscr{M}_{g}^{0}:=\left(\mathscr{M}^{0} / I_{g}\right) \otimes_{\mathbb{T}} \mathcal{O}_{g, \mathfrak{m}}, \quad \mathscr{M}_{g}:=\left(\mathscr{M} / I_{g}\right) \otimes_{\mathbb{T}} \mathcal{O}_{g, \mathfrak{m}} .
$$


Proposition 4.1. The natural map $\mathscr{M}_{g}^{0} \rightarrow \mathscr{M}_{g}$ is an isomorphism and $\mathscr{M}_{g}^{0}$ is free of rank one over $\mathcal{O}_{g, \mathfrak{m}}$.

Proof. The first part follows by tensoring the exact sequence induced by the degree map with $\mathcal{O}_{g, \mathfrak{m}}$ and using the fact that the Hecke action on $\mathscr{M} / \mathscr{M}^{0}=\mathbb{Z}$ is Eisenstein; the second is a consequence of Lemma 3.1plus Theorem 2.8. Details on this circle of ideas can be found in [20, $§ 2.1]$.

4.2. Algebraic parts of special values. Recall that an embedding $\psi: K \hookrightarrow \mathcal{B}$ is an optimal embedding of $\mathcal{O}_{K}$ into an Eichler order $R$ of $\mathcal{B}$ if $\psi^{-1}(R)=\mathcal{O}_{K}$. Fix an orientation (in the sense of $[2, \S 2.2]$ ) of $\mathcal{O}_{K}$ and let $h:=\# \operatorname{Pic}\left(\mathcal{O}_{K}\right)$ be the class number of $K$. There are exactly $h$ distinct conjugacy classes $\left[\psi_{1}\right], \ldots,\left[\psi_{h}\right]$ of oriented optimal embeddings of $\mathcal{O}_{K}$ into some oriented Eichler order of level $M$ of $\mathcal{B}$, which correspond to Gross-Heegner points of conductor 1; in fact, there is a simply transitive action of $\operatorname{Pic}\left(\mathcal{O}_{K}\right)$ on the $\left[\psi_{j}\right]$ (cf. [3, §1] and the references therein; in particular, see [2, §2.3] and [11, §3]). Each such conjugacy class $\left[\psi_{j}: \mathcal{O}_{K} \hookrightarrow R_{\psi_{j}}\right]$ gives rise to the element $\left[R_{\psi_{j}}\right] \in \mathcal{E}$, and we define

$$
\psi_{K}:=\left[R_{\psi_{1}}\right]+\cdots+\left[R_{\psi_{h}}\right] \in \mathscr{M} .
$$

Definition 4.2. The algebraic part $\mathbb{L}_{K}(g, 1)$ of $L_{K}(g, 1)$ is the image of $\psi_{K}$ in the rank one $\mathcal{O}_{g, \mathfrak{m}}$-module $\mathscr{M}_{g}^{0}=\mathscr{M}_{g}$.

This definition is justified by the following result.

Theorem 4.3. $L_{K}(g, 1)=0$ if and only if $\mathbb{L}_{K}(g, 1)=0$.

Proof. Let $\psi_{K, g}$ denote the projection of $\psi_{K}$ on the $g$-isotypic component of $\mathscr{M} \otimes$ $\mathbb{C}$. By [3, Theorem 1.1], the special value $L_{K}(g, 1)$ is a non-zero multiple of $\left\langle\psi_{K, g}, \psi_{K, g}\right\rangle$, where $\langle\cdot, \cdot\rangle$ is the positive-definite scalar product on $\mathscr{M}$ described in [3. §1]. Since $\psi_{K, g}=0$ if and only if $\mathbb{L}_{K}(g, 1)=0$, the result follows.

4.3. Heegner points on Shimura curves. As before, let $h$ be the class number of $K$ and write $P_{1}, \ldots, P_{h}$ for the Heegner points of conductor 1 on $X_{0}^{D}(M)$, as defined in [2, §2.1]; these points are permuted transitively by $\operatorname{Gal}(H / K)$ (see [2, §2.3]). Analogously, let $\mathcal{P}_{1}, \ldots, \mathcal{P}_{h}$ be the Heegner points of conductor 1 on $X_{0}^{D}(M \ell)$. Replacing $\mathcal{O}_{K}$ with the order of $K$ of conductor $\ell$, one can also consider Heegner points of conductor $\ell$ on $X_{0}^{D}(M \ell)$, which are rational over $\mathcal{L}$. Since $\ell$ is inert in $K$, there are exactly $h(\ell+1) / u$ such points, to be denoted $\mathcal{P}_{i}^{\prime}$, on which $\operatorname{Gal}(\mathcal{L} / K)$ acts simply transitively ([2, Lemma 2.5]). For more details and proofs, see [2, Section $2]$.

Let $N_{\mathcal{L} / H}$ be the norm map. As explained in [2, §2.4], we can (and do) choose orientations of the quadratic orders and of the Eichler orders in such a way that (up to renumbering) for every $i=1, \ldots, h$ there is an equality $u N_{\mathcal{L} / H}\left(\mathcal{P}_{i}^{\prime}\right)=\mathcal{P}_{i}$ of divisors on $X_{0}^{D}(M \ell)$. Now define

$$
P_{K}:=\sum_{i=1}^{h} \iota_{M}\left(P_{i}\right) \in J_{0}^{D}(M)(K), \quad \mathcal{P}_{\mathcal{L}}^{\prime}:=\sum_{i=1}^{h} \iota_{M \ell}\left(\mathcal{P}_{i}^{\prime}\right) \in J_{0}^{D}(M \ell)(\mathcal{L}) .
$$

Having the points $\mathcal{P}_{i}$ at our disposal, fix the orientation of $R(M)$ so that $\pi_{1}\left(\mathcal{P}_{i}\right)=P_{i}$ for all $i=1, \ldots, h$. Thanks to the commutativity of square (5),

$$
P_{K}=u \pi_{1, *}\left(N_{\mathcal{L} / H}\left(\mathcal{P}_{\mathcal{L}}^{\prime}\right)\right) .
$$


Finally, define the Heegner point

$$
\alpha_{K}:=\Pi_{E}\left(P_{K}\right) \in E(K) .
$$

This is the point in terms of which we shall state our main result.

4.4. Jochnowitz congruences and main result. Recall the isomorphism $\eta$ defined in (9) and let $\tilde{\pi}_{J}: J_{0}^{D}(M \ell)\left(M_{\ell}\right) \rightarrow \tilde{J} / \mathfrak{m} \tilde{J}$ be the map induced by $\pi_{J}$. Before stating our main result (Theorem 4.6), we need two lemmas.

Lemma 4.4. $u \operatorname{deg}\left(\pi_{E}\right) \cdot \boldsymbol{n}\left(\tilde{\pi}_{J}\left(\mathcal{P}_{\mathcal{L}}^{\prime}\right)\right)=\boldsymbol{i}\left(\left(\alpha_{K}, 0\right)\right)$.

Proof. Adopt the symbol $[\star]$ to denote the class modulo $\mathfrak{m}$ of an element $\star$. Using (10), the functoriality of the Hodge class (cf. [8, eq. (3.7)]) and the fact that the Hecke action on Hodge classes is Eisenstein, it can be checked that the equality $u \cdot \boldsymbol{n}\left(\tilde{\pi}_{J}\left(\mathcal{P}_{\mathcal{L}}^{\prime}\right)\right)=\left[\pi_{J}\left(\pi_{1}^{*}\left(P_{K}\right)\right)\right]$ holds in $J\left(K_{\ell}\right) / \mathfrak{m} J\left(K_{\ell}\right)$. The claim then follows as in the proof of [5, Lemma 6.3].

Lemma 4.5. $\boldsymbol{p}\left(\tilde{\pi}_{J}\left(\mathcal{P}_{\mathcal{L}}^{\prime}\right)\right)=\boldsymbol{j}\left(\mathbb{L}_{K}(g, 1)\right)$.

Proof. This is a consequence of [3, Theorem 3.2].

Write $\left[\mathbb{L}_{K}(g, 1)\right]$ for the class of $\mathbb{L}_{K}(g, 1)$ in $\mathscr{M}_{g}^{0} / \mathfrak{m}_{g} \mathscr{M}_{g}^{0}=\mathscr{M}^{0} / \mathfrak{m} \mathscr{M}^{0}$.

Theorem 4.6. If $g$ is an eigenform on $J^{\prime}$, then the equality

$$
\eta\left(\left(\alpha_{K}, 0\right)\right)=u \operatorname{deg}\left(\pi_{E}\right) \cdot\left[\mathbb{L}_{K}(g, 1)\right]
$$

holds in $\mathscr{M}^{0} / \mathfrak{m} \mathscr{M}^{0}$.

Proof. Using Lemmas 4.4 and 4.5, proceed as in the proof of [5, Theorem 6.1].

Theorem 4.6 immediately implies Theorem 1.1 .

Remark 4.7. By the formula of Gross-Zagier type proved by Zhang in 21, Theorem $\mathrm{C}]$, the point $\alpha_{K}$ encodes, via its Néron-Tate height, the central derivative $L_{K}^{\prime}(E, 1)$. Hence Theorem 4.6 (or, rather, Theorem 1.1) can be viewed as providing a congruence modulo $\mathfrak{m}$ between $L_{K}^{\prime}(E, 1)$ and $L_{K}(g, 1)$.

4.5. Some consequences. We collect two consequences of our main result.

Proposition 4.8. If the image of $\alpha_{K}$ in $E\left(K_{\ell}\right) / p E\left(K_{\ell}\right)$ is non-zero, then $L_{K}\left(J^{\prime}, 1\right) \neq 0$.

Proof. By Theorem 1.1 $\left[\mathbb{L}_{K}(g, 1)\right] \neq 0$ for all eigenforms $g$ on $J^{\prime}$; hence $L_{K}(g, 1) \neq$ 0 by Theorem 4.3. But $L_{K}\left(J^{\prime}, 1\right)=\prod_{g} L_{K}(g, 1)$, where the product is taken over the distinct eigenforms on $J^{\prime}$, and the claim follows.

For the proof of the following proposition see [5, Proposition 1.5].

Proposition 4.9. If the image of $\alpha_{K}$ in $E\left(K_{\ell}\right) / p E\left(K_{\ell}\right)$ is non-zero, then

(1) the $p$-Selmer group of $E$ over $K$ is one-dimensional over $\mathbb{F}_{p}$ and is generated by the image of $\alpha_{K}$ under the Kummer map;

(2) the $\mathfrak{m}$-Selmer group of $J^{\prime}$ is trivial; hence $J^{\prime}(K)$ is finite.

For arithmetic results in the same vein as Proposition 4.9] establishing a link between the local $p$-divisibility of the Heegner point $\alpha_{K}$ at the prime $\ell$ and the $p$ descent on a related abelian variety of level $N \ell$, the reader is referred to the paper 12. by Gross and Parson. 


\section{ACKNOWLEDGEMENTS}

The author would like to thank the anonymous referee for several valuable remarks and suggestions that led to significant improvements in the exposition.

\section{REFERENCES}

[1] Amod Agashe, Kenneth A. Ribet, and William A. Stein, The modular degree, congruence primes, and multiplicity one, Number theory, analysis and geometry, Springer, New York, 2012, pp. 19-49, DOI 10.1007/978-1-4614-1260-1_2. MR2867910

[2] M. Bertolini and H. Darmon, Heegner points on Mumford-Tate curves, Invent. Math. 126 (1996), no. 3, 413-456, DOI 10.1007/s002220050105. MR:1419003 (97k:11100)

[3] M. Bertolini and H. Darmon, A rigid analytic Gross-Zagier formula and arithmetic applications, Ann. of Math. (2) 146 (1997), no. 1, 111-147, DOI 10.2307/2951833. with an appendix by Bas Edixhoven. MR 1469318 (99f:11079)

[4] M. Bertolini and H. Darmon, Heegner points, p-adic L-functions, and the Cerednik-Drinfeld uniformization, Invent. Math. 131 (1998), no. 3, 453-491, DOI 10.1007/s002220050211. MR.1614543(99f:11080)

[5] M. Bertolini and H. Darmon, Euler systems and Jochnowitz congruences, Amer. J. Math. 121 (1999), no. 2, 259-281. MR1680333 (2001d:11060)

[6] M. Bertolini and H. Darmon, Iwasawa's main conjecture for elliptic curves over anticyclotomic $\mathbb{Z}_{p}$-extensions, Ann. of Math. (2) 162 (2005), no. 1, 1-64, DOI 10.4007/annals.2005.162.1. MR2178960 (2006g:11218)

[7] Pete L. Clark, On the Hasse principle for Shimura curves, Israel J. Math. 171 (2009), 349365, DOI 10.1007/s11856-009-0053-6. MR2520114 (2010k:11097)

[8] Christophe Cornut and Vinayak Vatsal, Nontriviality of Rankin-Selberg L-functions and $C M$ points, L-functions and Galois representations, London Math. Soc. Lecture Note Ser., vol. 320, Cambridge Univ. Press, Cambridge, 2007, pp. 121-186, DOI 10.1017/CBO9780511721267.005. MR2392354 (2009m:11088)

[9] Henri Darmon, Rational points on modular elliptic curves, CBMS Regional Conference Series in Mathematics, vol. 101, published for the Conference Board of the Mathematical Sciences, Washington, DC, by the Amer. Math. Soc., Providence, RI, 2004. MR2020572 (2004k:11103)

[10] Fred Diamond and Richard Taylor, Nonoptimal levels of mod $l$ modular representations, Invent. Math. 115 (1994), no. 3, 435-462, DOI 10.1007/BF01231768. MR1262939(95c:11060)

[11] Benedict H. Gross, Heights and the special values of L-series, Number theory (Montreal, Que., 1985), CMS Conf. Proc., vol. 7, Amer. Math. Soc., Providence, RI, 1987, pp. 115-187. MR.894322(89c:11082)

[12] Benedict H. Gross and James A. Parson, On the local divisibility of Heegner points, Number theory, analysis and geometry, Springer, New York, 2012, pp. 215-241, DOI 10.1007/978-14614-1260-1_11. MR2867919

[13] David Helm, On maps between modular Jacobians and Jacobians of Shimura curves, Israel J. Math. 160 (2007), 61-117, DOI 10.1007/s11856-007-0056-0. MR2342491 (2009e:11119)

[14] Naomi Jochnowitz, A p-adic conjecture about derivatives of L-series attached to modular forms (Boston, MA, 1991), Contemp. Math., vol. 165, Amer. Math. Soc., Providence, RI, 1994, pp. 239-263, DOI 10.1090/conm/165/01608. MR1279612 (95g:11037)

[15] San Ling, Shimura subgroups and degeneracy maps, J. Number Theory 54 (1995), no. 1, 39-59, DOI 10.1006/jnth.1995.1100. MR1352635 (96h:11058)

[16] B. Mazur, Modular curves and the Eisenstein ideal, Inst. Hautes Études Sci. Publ. Math. 47 (1977), 33-186 (1978). MR488287(80c:14015)

[17] K. A. Ribet, On modular representations of $\mathrm{Gal}(\overline{\mathbf{Q}} / \mathbf{Q})$ arising from modular forms, Invent. Math. 100 (1990), no. 2, 431-476, DOI 10.1007/BF01231195. MR1047143 (91g:11066)

[18] David Peter Roberts, Shimura curves analogous to $X_{0}(N)$, Thesis (Ph.D.)-Harvard University, 1989, ProQuest LLC, Ann Arbor, MI. MR2637583

[19] Jean-Pierre Serre, Propriétés galoisiennes des points d'ordre fini des courbes elliptiques (French), Invent. Math. 15 (1972), no. 4, 259-331. MR0387283 (52 \#8126)

[20] V. Vatsal, Special values of anticyclotomic L-functions, Duke Math. J. 116 (2003), no. 2, 219-261, DOI 10.1215/S0012-7094-03-11622-1. MR1953292(2004e:11069b) 
[21] Shouwu Zhang, Heights of Heegner points on Shimura curves, Ann. of Math. (2) 153 (2001), no. 1, 27-147, DOI 10.2307/2661372. MR1826411 (2002g:11081)

[22] Shou-Wu Zhang, Gross-Zagier formula for GL2, Asian J. Math. 5 (2001), no. 2, 183-290. MR:1868935 (2003k:11101)

Dipartimento di Matematica, Università di Genova, Via Dodecaneso 35, 16146 Genova, ITALY

E-mail address: vigni@dima.unige.it 